\title{
CT Systems
}

\author{
Thomas Flohr
}

Published online: 9 January 2013

(C) Springer Science+Business Media New York 2013

\begin{abstract}
Computed tomography (CT) was introduced in the early 1970s, and has since then revolutionized diagnostic imaging. Today, CT is the backbone of radiology. In this article we review different CT system design concepts. We start with an overview of the "classic" four generations of CT systems: the first generation head scanners relying on the "translate-rotate" principle, second generation scanners with a small detector array instead of a single detector, modern third generation "rotate-rotate" CT scanners with a detector fan wide enough to cover a whole-body scan field of view, and finally the currently abandoned fourth generation CT systems with a rotating X-ray tube and stationary detector. We present basic concepts and a short history of multidetector row CT (MDCT), illustrating its potential and limitations. Then, we introduce novel system concepts, such as CT systems with area detectors and dual source CT, that aim at solving remaining limitations of MDCT. We explain the basic system options to acquire dual energy CT data or spectrally resolved CT data. Finally, we briefly touch alternative system designs, such as electron beam CT, CT systems with inverse geometry, interior tomography $\mathrm{CT}$, and phase contrast CT.
\end{abstract}

Keywords Computed tomography - CT systems . Multi-detector row CT $\cdot$ Dual source CT $\cdot$ EBCT

T. Flohr ( $\square)$

Siemens Healthcare, Computed Tomography,

Siemensstr. 1, 91301 Forchheim, Germany

e-mail: thomas.flohr@siemens.com

T. Flohr

Department of Diagnostic Radiology, Eberhard Karls University, Hoppe-Seyler-Str. 3, 72076 Tübingen, Germany

\section{Introduction}

Computed tomography (CT) was introduced in the early 1970s and has since then revolutionized diagnostic radiology. It was the first medical imaging machine to use a computer, in this way pioneering the era of digital imaging. CT technology has significantly improved and matured in the past 40 years. Today, CT is the backbone of radiology. In this article we review the different generations of CT system designs, from the first dedicated head scanners to modern multi detector row CTs (MDCT). We introduce novel system concepts, such as CT systems with area detectors and dual source CTs, that aim at solving remaining limitations of MDCT. We explain the basic system options to acquire dual energy CT data or spectrally resolved CT data. Finally, we briefly touch alternative system designs such as electron beam CT (EBCT), CT systems with inverse geometry, interior tomography $\mathrm{CT}$ or phase contrast $\mathrm{CT}$.

\section{Basic Principles of CT}

In X-ray CT, local X-ray attenuation coefficients are measured in a slice of the patient's anatomy. They are translated into grey-scale values and displayed in the CT image, usually with a matrix of 512 by 512 pixels. To compute the local $\mathrm{X}$-ray attenuation coefficients, the patient's X-ray absorption is measured along narrow X-ray beams in many different directions within the desired image plane. A detector records the attenuated intensity of each transmitted X-ray beam after it has passed through the patient's body-the logarithm of the attenuated intensity is the line-integral of the patient's attenuation coefficients along the beam path. Multiple line integrals across an object can be acquired by the translation of a narrow X-ray beam and detector system, or a broader, 
fan-shaped beam and multiple detector elements across the object (see Fig. 1). The combination of line integrals that fully encompass the object along the same angular position of the measurement system comprises one view, or projection. To allow mathematical reconstruction of the attenuation coefficients in a slice of the object, multiple projections at different angles covering half a rotation of the measurement system around the patient have to be acquired-fan beam geometry needs $180^{\circ}$ of data plus the total fan angle of the detector. Today, about 1,000 projections are measured in a full $360^{\circ}$ rotation, each comprising about 700-900 individual measurement values (line integrals).

\section{Different Generations of CT Systems}

Since its clinical introduction in the early 1970s, the technology used in CT scanners has matured significantly. Figure 1 illustrates the geometries of the first four generations of X-ray CT scanners.

First-generation CT systems, starting with Sir Godfrey Hounsfield's original head scanner, the EMI Mark I, were based on the "translate-rotate" principle [1]. These employed a narrow pencil X-ray beam and two detectors that simultaneously acquired two views in the through-plane direction (the through-plane direction or z-direction is along the patient's longitudinal axis). The measurement values of each projection were acquired sequentially by a linear shift of $\mathrm{X}$-ray tube and detector, followed by a rotation of the measurement system by a small angle $\left(1^{\circ}\right)$ to measure the next projection. Figure 2 shows the Siemens head scanner SIRETOM 1, which was quite similar to the EMI Mark I. The narrow-beam geometry required significantly long scan times (5-7 min for two simultaneously acquired anatomic levels). The image was displayed with an 80 by 80 matrix. The scan field of view (SFOV) had a diameter of $25 \mathrm{~cm}$-the system was therefore limited to head scans. Spatial resolution was $1.3 \mathrm{~mm}$ (about four line pairs per $\mathrm{cm}$ ). Despite that these are "poor" image quality parameters from today's perspective, the first CT images revealed the patient's anatomy with a then unknown differentiation of low-contrast details, see Fig. 2.

The next, second-generation CT systems were based on the same "translate-rotate" principle, but the single detector was replaced by an array of about 30 detectors that covered a fan angle of approximately $10^{\circ}$. As a consequence, the acquisition time for a slice could be reduced to $18 \mathrm{~s}$. Secondgeneration CT scanners were single-slice systems. Multiple detectors in the through-plane direction, as already implemented in the first CT scanner generation, were eliminated from most scanner designs until the recent resurgence of "multi-slice" scanning in the mid 1990s.

The most common CT systems today are third-generation scanners. They employ the so-called "rotate-rotate" geometry, in which both the X-ray tube and a detector array rotate about the patient (see Fig. 3). The fan angle of the detector is wide enough (approximately $45^{\circ}-55^{\circ}$ ) to cover a whole-body SFOV with a diameter of $50 \mathrm{~cm}$-in this way, routine body scanning became feasible. A projection was no longer built-up by sequential measurements requiring a translation of detector and X-ray tube, but was instead simultaneously acquired by 700-900 individual detectors in the fan. This way, scan times per slice dropped rapidly. As a next step, slip-ring designs which passed the electrical signals across sliding contacts allowed for continuous rotation of the measurement system and eliminated the need to rewind the gantry after each rotation in order to avoid the tangling of cables. Using this technology, rotation times of
Fig. 1 Design and acquisition schemes of four generations of X-ray CT scanners
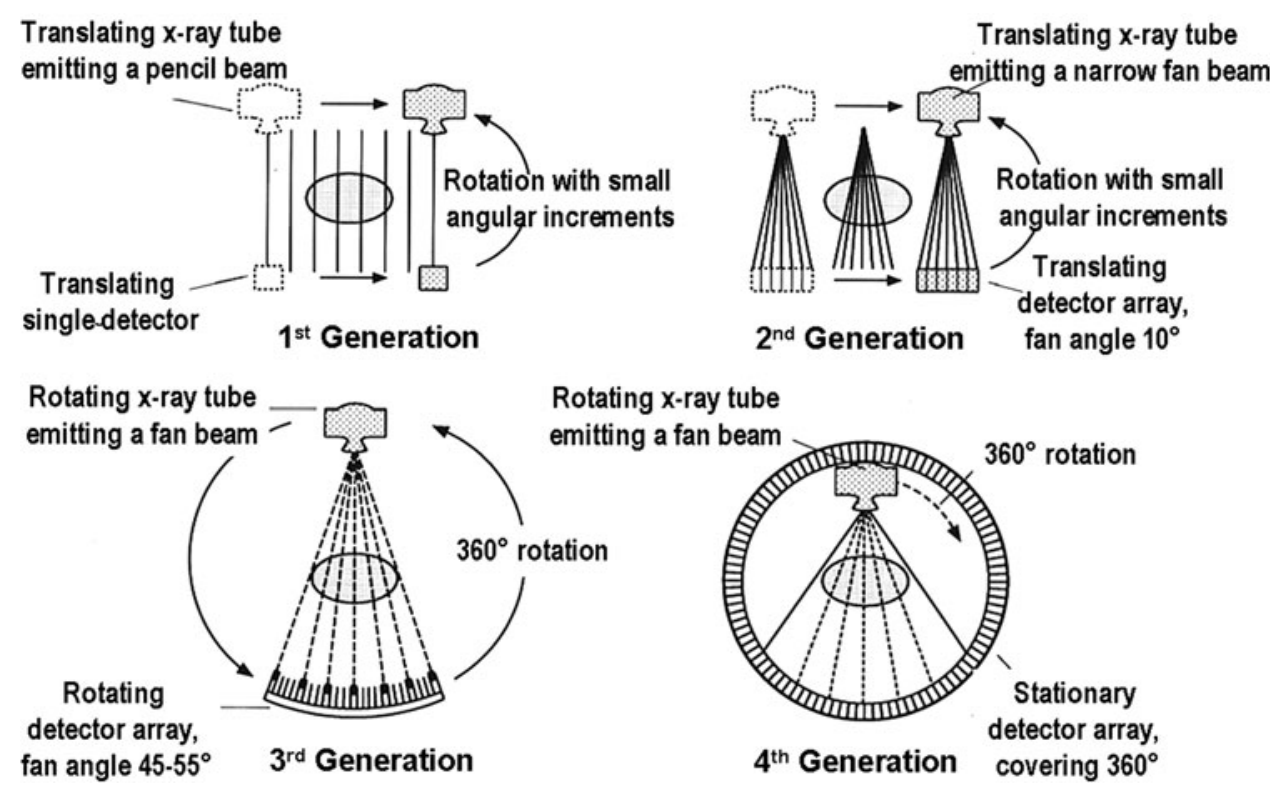

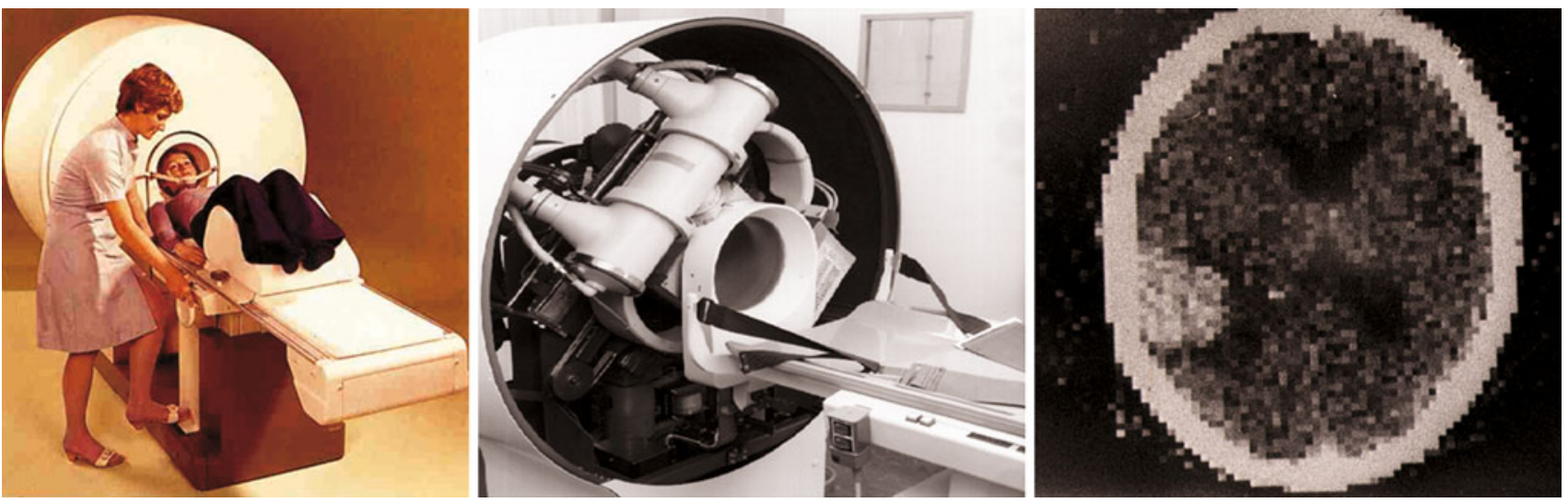

Fig. 2 A first-generation CT head scanner, the Siemens SIRETOM 1, and a head image acquired with this scanner

less than a second were achieved in the early 1990s. In 2012, the fastest CT scanner needed $0.27 \mathrm{~s}$ for a $360^{\circ}$ rotation of the tube and detector system. Slip-ring gantries were also a prerequisite for spiral (helical) CT scanning with continuous data acquisition and continuous table movement, which has revolutionized medical CT since 1990 [2, 3••]. With the use of spiral CT, entire organs, such as lung or liver, could be examined within one breath-hold and without the danger of mis- or double-registration of anatomical details.

A fourth-generation of CT systems utilized a wide fan beam and a rotating X-ray tube, but a stationary array of detectors that encircled the patient. These systems had fewer moving parts and needed not pass signal data across the moving gantry. They had the potential to enable very

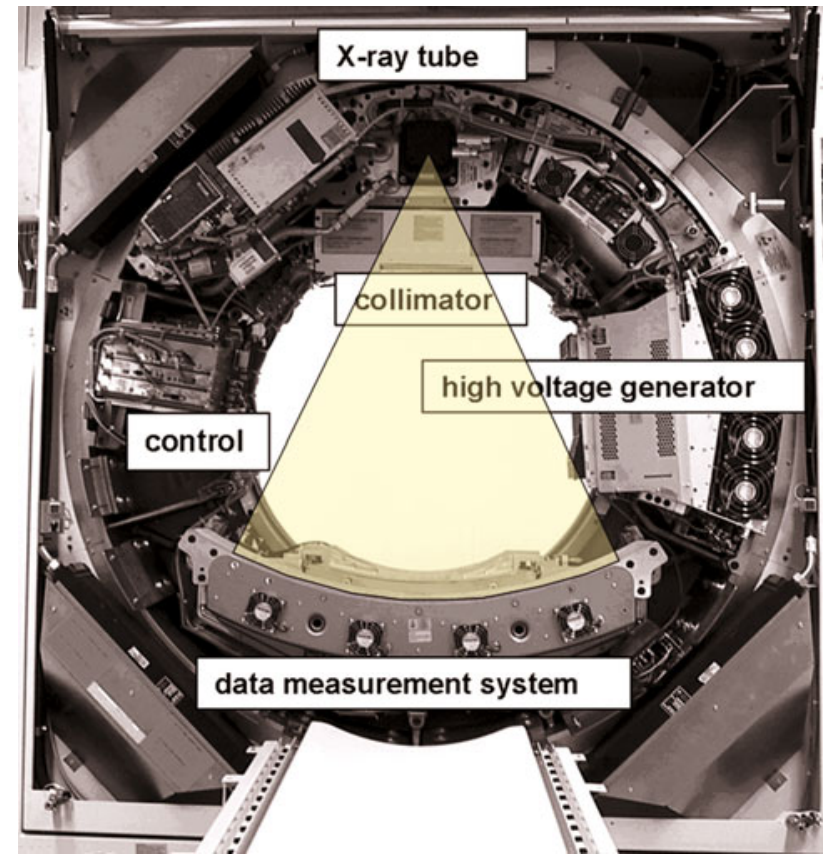

Fig. 3 Components of a modern third generation CT system. The fan beam covering a whole-body scan field of view with a diameter of $50 \mathrm{~cm}$ is indicated fast rotation of the X-ray tube around the patient, and therefore require very short acquisition times per slice. The design principle, however, has currently been abandoned because of high system costs as a consequence of the large number of detector elements and other problems. As an example, focused post-patient collimators (near the detector surface) to suppress scattered radiation could not be used, and the images suffered from severe scatter artifacts.

\section{Multi-Detector Row CT}

Since the late 1990s, MDCT systems based on the third generation rotate-rotate geometry have dominated the CT market. The most important advance in these CT scanners is the implementation of X-ray detectors that are physically separated in the $z$-axis direction and enable simultaneous acquisition of multiple slices of the patient's anatomy. Key benefits of MDCT systems are faster scan speed, improved through-plane resolution and better utilization of the available X-ray tube power. MDCT also expanded into new clinical areas, such as CT angiography of the coronary arteries with the addition of ECG gating capability $[4,5]$.

To provide slices at different collimated slice widths, detectors with a larger number of detector rows than finally read-out slices are used. The total beam width in the $z$-direction is adjusted by pre-patient collimation, and the signals of every two (or more) detectors along the $z$-axis are electronically combined to thicker slices, see Fig. 4.

The first MDCT systems introduced in 1998 offered simultaneous acquisition of four slices with a thinnest collimated slice width of 1 or $1.25 \mathrm{~mm}$ at a rotation time of down to $0.5 \mathrm{~s}$ [6-8]. An 8-slice CT system, introduced in 2000, enabled shorter examination times but no improved spatial resolution (thinnest collimation $8 \times 1.25 \mathrm{~mm}$ ). In 2001, 16-slice CT systems [9] became commercially available, with collimations of $16 \times 0.5,16 \times 0.625$ or $16 \times 0.75 \mathrm{~mm}$ and faster gantry rotation (down to $0.42 \mathrm{~s}$ and later $0.375 \mathrm{~s}$ ). 
Fig. 4 Multidetector row CT system utilizing a detector with 24 detector rows to provide 16 slices at different slice widths. If only the central 16 rows are illuminated, they are read-out individually, and the detector provides 16 slices with, e.g., $0.625-$ or $0.75-\mathrm{mm}$ slice width $(4$, top $)$. To obtain 16 collimated 1.25 - or $1.5-\mathrm{mm}$ slices, the prepatient collimator is opened. The full $z$-width of the detector is illuminated, and the signals of every 2 central rows are electronically combined (4, bottom)
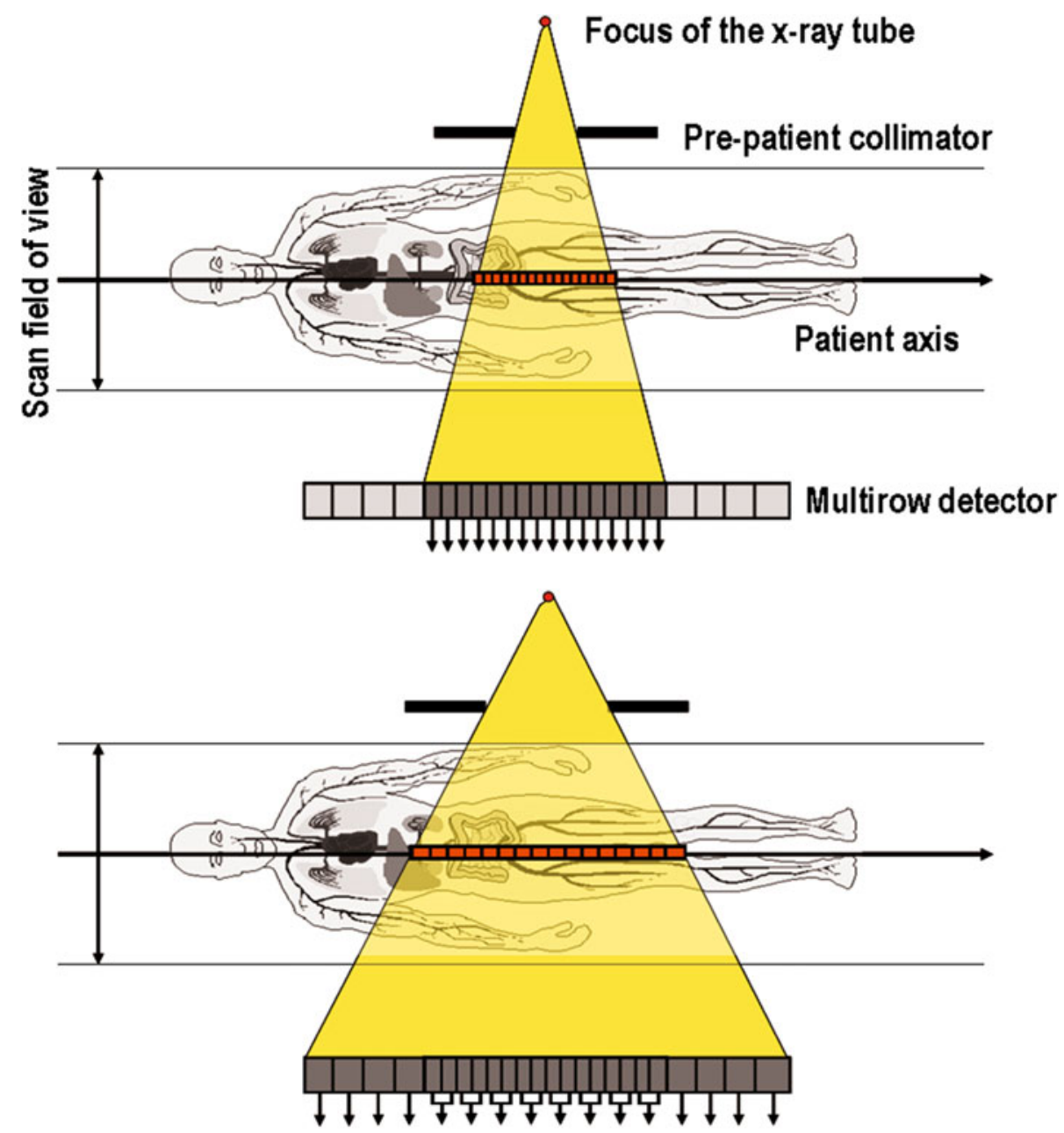

In 2004, all major CT manufacturers introduced MDCT systems with simultaneous acquisition of 64-slices at 0.5-, $0.6-$ or $0.625-\mathrm{mm}$ collimated slice width, and further reduced rotation times (down to $0.33 \mathrm{~s}$ ). GE, Philips and Toshiba aimed at increasing volume coverage speed by using detectors with 64 rows, in this way providing 64 collimated $0.5-\mathrm{mm}$ or $0.625-\mathrm{mm}$ slices with a total $z$-coverage of 32 or $40 \mathrm{~mm}$. Siemens used 32 physical detector rows in combination with a $z$-flying focal spot to simultaneously acquire 64 overlapping $0.6-\mathrm{mm}$ slices with a total $z$-coverage of $19.2 \mathrm{~mm}$, with the goal of pitch-independent increase of through-plane resolution and reduction of spiral artifacts [10]. With 64-slice CT systems CT scans with isotropic sub-mm resolution became feasible even for extended anatomical ranges (see Fig. 5).

The improved temporal resolution due to faster gantry rotation increased clinical robustness of ECG-gated scanning, thereby facilitating the successful integration of CT coronary angiography into routine clinical algorithms [11,12], although higher and irregular heart rates were still problematic.

In 2007, one vendor introduced a MDCT system with 128 simultaneously acquired slices, based on a 64-row detector with $0.6-\mathrm{mm}$ collimated slice width and $z$-flying focal spot. Recently, simultaneous acquisition of 256 slices became available, with a CT system equipped with a 128-row detector (0.625-mm collimated slice width) and $z$-flying focal spot.

Clinical experience with 64-, 128- or 256-slice CT indicates that the performance level of MDCT has reached a level of saturation, and mere adding of even more detector rows will not by itself translate into increased clinical benefit. Instead, developments are ongoing to solve remaining limitations of MDCT.

\section{CT Systems with Area Detector}

One remaining challenge for MDCT is the visualization of dynamic processes in extended anatomical ranges, e.g., to characterize the inflow and outflow of contrast agent in the arterial and venous system in dynamic CT angiographic studies (CTAs), or to determine the enhancement characteristics of the contrast agent in volume perfusion scans. One way to solve this problem is the introduction of area 
Fig. 5 Whole body CT angiographic scan acquired with a 64-slice CT system at submillimeter isotropic spatial resolution. The total scan time with 0.5 -s gantry rotation was less than $30 \mathrm{~s}$ (courtesy of Eberhard Karls University, Tübingen, Germany)

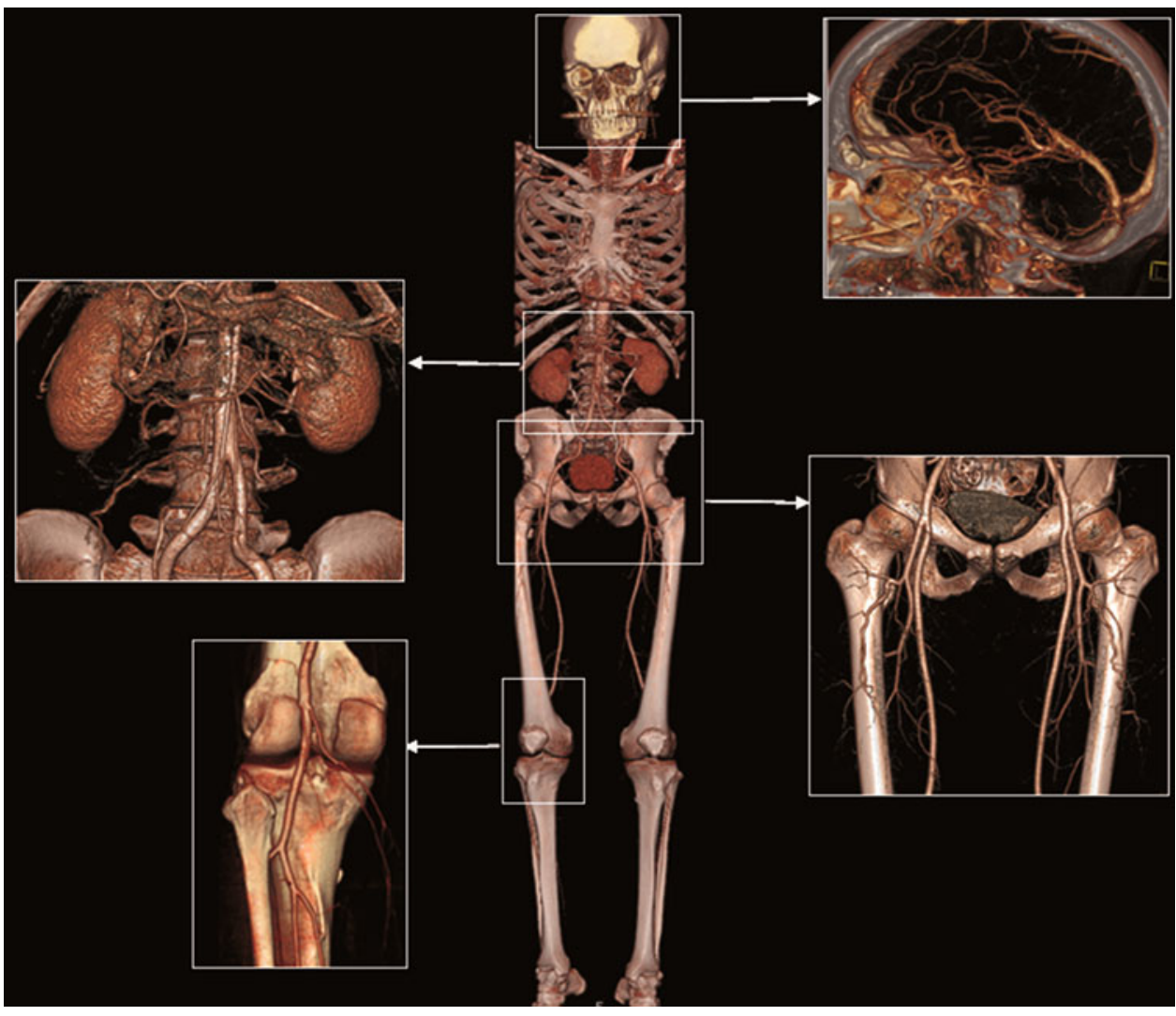

detectors large enough to cover entire organs, such as the heart, the kidneys or the brain, in one axial scan.

In 2007 a CT-scanner with $320 \times 0.5-\mathrm{mm}$ collimation, covering $16 \mathrm{~cm}$ at iso-center, and 0.35 -s gantry rotation time was commercially introduced by one vendor, after a long evaluation phase using prototype systems (see Fig. 6) with $256 \times 0.5-\mathrm{mm}$ collimation and 0.5 -s gantry rotation time [13-16].

Computed tomography scanners with area detector have advantages in cardiac scanning and in the acquisition of dynamic CT data. Figure 7 shows a clinical example for a pediatric cardiovascular CT examination with a 320-row CT system.

With the typical MDCT detector $z$-coverages of $40 \mathrm{~mm}$ (and recently up to $80 \mathrm{~mm}$ ), ECG-controlled CT volume imaging of the heart is comprised of several sub-volumes acquired during two to four consecutive heart beats. CT systems with large area detectors can image the entire heart in one axial scan without table movement. Meanwhile, successful use of the commercially available CT system with $320 \times 0.5-\mathrm{mm}$ detector collimation for coronary CTA has been demonstrated [17-19].

As a second benefit, CT systems with area detectors can acquire dynamic volume data by repeatedly scanning the same anatomical range without table movement, which is useful in dynamic CTAs or in volume perfusion studies, e.g., in the management of stroke patients or in oncology [20], or for the evaluation of myocardial perfusion defects.

A challenge of larger detector $z$-coverage in particular for perfusion scanning is increased X-ray scatter. Scattered radiation may cause hypodense artifacts, may affect CTnumber stability, and the scatter induced noise may reduce the contrast-to-noise-ratio (CNR) in the images [21]. The

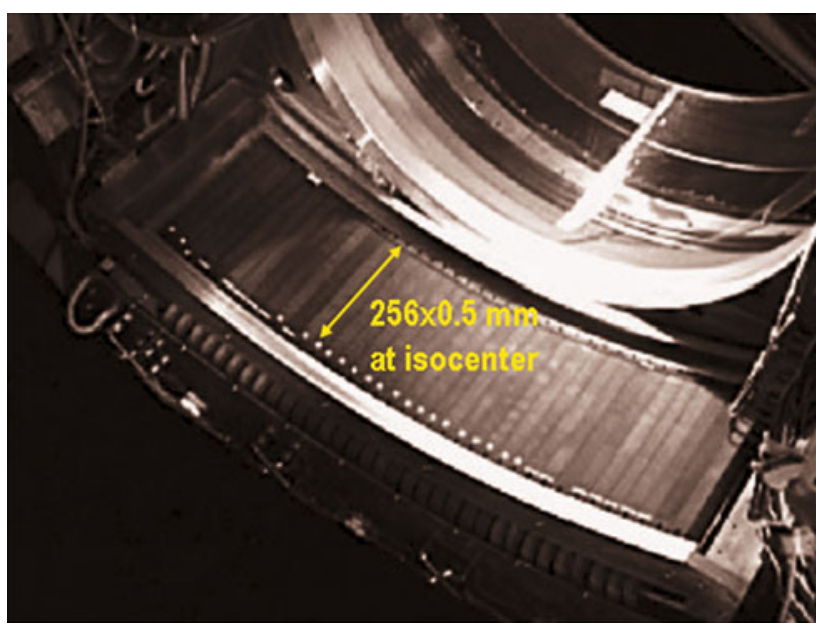

Fig. 6 Prototype of a CT area detector providing 256 collimated 0.5 -mm slices. From [52], with permission 

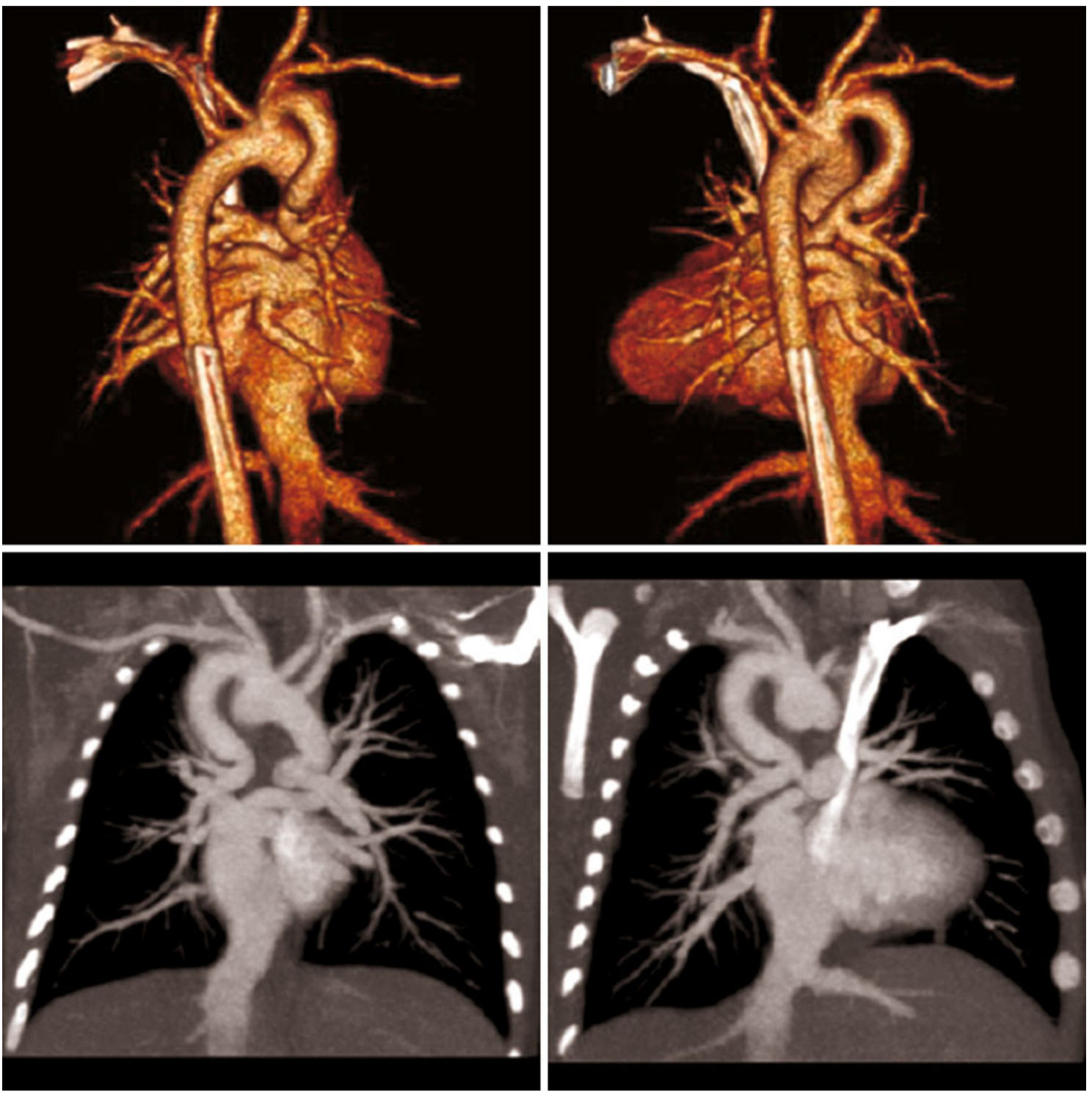

Fig. 7 Congenital anomaly in a 4-day old boy. The pulmonary arteries arise from the aortic arch. The pulmonary veins drain normally into the left atrium. The scan was performed in a single

magnitude of scatter artifacts scales linearly with the illuminated $z$-width of the detector [22].

An alternative approach to dynamic CT scanning of a larger volume is the use of a spiral shuttle mode. By periodically moving the patient forward and backward during data acquisition, a scan range larger than the detector $z$-width is covered. The scan range can be flexibly adapted to the organ of interest. As a downside, the maximum temporal sampling rate is lower than with a wide detector, and data sampling is temporally equidistant only in the center of the scanned area. The non-equidistant data sampling at different $z$-positions within the volume has to be considered in the calculation of the perfusion parameters; their accuracy, however, is not compromised [23]. 0.35-s gantry rotation using a CT scanner with $320 \times 0.5-\mathrm{mm}$ collimation (courtesy of R. Mather, Toshiba Medical Systems, USA)

\section{Dual Source CT}

Another remaining challenge for MDCT is insufficient temporal resolution for cardiac CT examinations, which require very short exposure time of the individual axial slices to freeze the motion of the heart. To improve temporal resolution in a clinically reliable way, the gantry has to rotate faster. Despite gantry rotation times of $0.3 \mathrm{~s}$ and less, motion artifacts at higher and irregular heart rates remain a challenge for coronary CTA. An alternative scanner concept that provides significantly improved temporal resolution but does not require faster gantry rotation is a CT with multiple tubes and corresponding detectors [24, 25]. In 2006, a dual source CT (DSCT), i.e., a CT 
scanner with two X-ray tubes and two corresponding detectors offset by $90^{\circ}$ [26] was commercially introduced by one vendor (see Fig. 8).

In 2009, the second generation of DSCT-systems was introduced, offering a larger SFOV of the B-detector $(33 \mathrm{~cm}$ instead of $26 \mathrm{~cm}$ ), wider $z$-coverage and faster gantry rotation.

The key benefit of DSCT for cardio-thoracic scanning is improved temporal resolution. In parallel geometry, $180^{\circ}$ of scan data (a half-scan sinogram) are necessary for image reconstruction. Due to the $90^{\circ}$ angle between both X-ray tubes, the half-scan sinogram can be split up into two $90^{\circ}$ data segments which are simultaneously acquired by the two acquisition systems in the same phase of the patient's cardiac cycle and at the same anatomical level. Therefore, the total data acquisition time per image is a quarter of the gantry rotation time $t_{\text {rot }} / 4$ in a sufficiently centered region of the SFOV [26]. For the first-generation DSCT with $t_{\text {rot }}=0.33 \mathrm{~s}$, the temporal resolution is $t_{\mathrm{rot}} / 4=83 \mathrm{~ms}$. For the secondgeneration DSCT with $t_{\text {rot }}=0.28 \mathrm{~s}$, it is $75 \mathrm{~ms}$ as a consequence of the increased system angle of $95^{\circ}$. Meanwhile, several clinical studies have demonstrated the potential of DSCT to provide diagnostic image quality in cardiac CT with little or no dependence on the patient's heart rate [27-31].

Dual source CT systems offer an alternative way to scan the heart within one heartbeat. With a single-source CT, the spiral pitch $p$ is limited to $p \leq 1.5$ to ensure gapless volume coverage along the $z$-axis; $p$ is defined as the table feed per rotation divided by the total detector $z$-coverage. If the pitch is increased beyond $p=1.5$, sampling gaps along the $z$-axis will lead to severe image artifacts. With DSCT systems, data acquired with the second measurement system a quarter rotation later can be used to fill these gaps. In this way, the pitch can be increased up to $p=3.4$ in a limited SFOV that is covered by both detectors $[32 \bullet, 33]$. Using this technique, the second generation DSCT can be operated at a speed of $458 \mathrm{~mm} / \mathrm{s}$, which is sufficient to cover the heart $(12 \mathrm{~cm})$ in about $0.27 \mathrm{~s}$. Each of the individual axial images has a temporal resolution of $75 \mathrm{~ms}$. The patient's ECG is used to trigger both table motion and data acquisition. Meanwhile, several clinical studies have demonstrated the successful use of the high-pitch scan technique for coronary CT angiography in patients with sufficiently low and stable heart rates at very low radiation doses $[34,35]$. Other potential applications of the high-pitch spiral are ECG-triggered scanning of the entire thorax at low radiation dose, see Fig. 9, or fast scanning of un-cooperative patients, such as in pediatric CT.

The main challenge for dual source CT is cross-scattered radiation, i.e., scattered radiation from $\mathrm{X}$-ray tube $\mathrm{B}$ detected by detector $\mathrm{A}$, and vice versa. Cross-scattered radiation can produce artefacts and degrade the CNR of the images, if not adequately corrected for.

Dual source CT systems show interesting properties for general radiology applications, too. Both X-ray tubes can be operated at different $\mathrm{kV}$ - and $\mathrm{mA}$-settings, allowing for the acquisition of dual energy data. The use of dual energy CT can in principle add functional information to the morphological information based on X-ray attenuation that is usually obtained in a CT examination.

\section{Dual Energy CT}

In CT, the two relevant interaction mechanisms of X-ray photons with matter are the photo effect and the Compton effect. Both show different dependence on the photon energy $E$ and on the atomic number $Z$ of the investigated materials. A material can therefore be identified by its characteristic change of attenuation when scanned with two different mean X-ray energies.
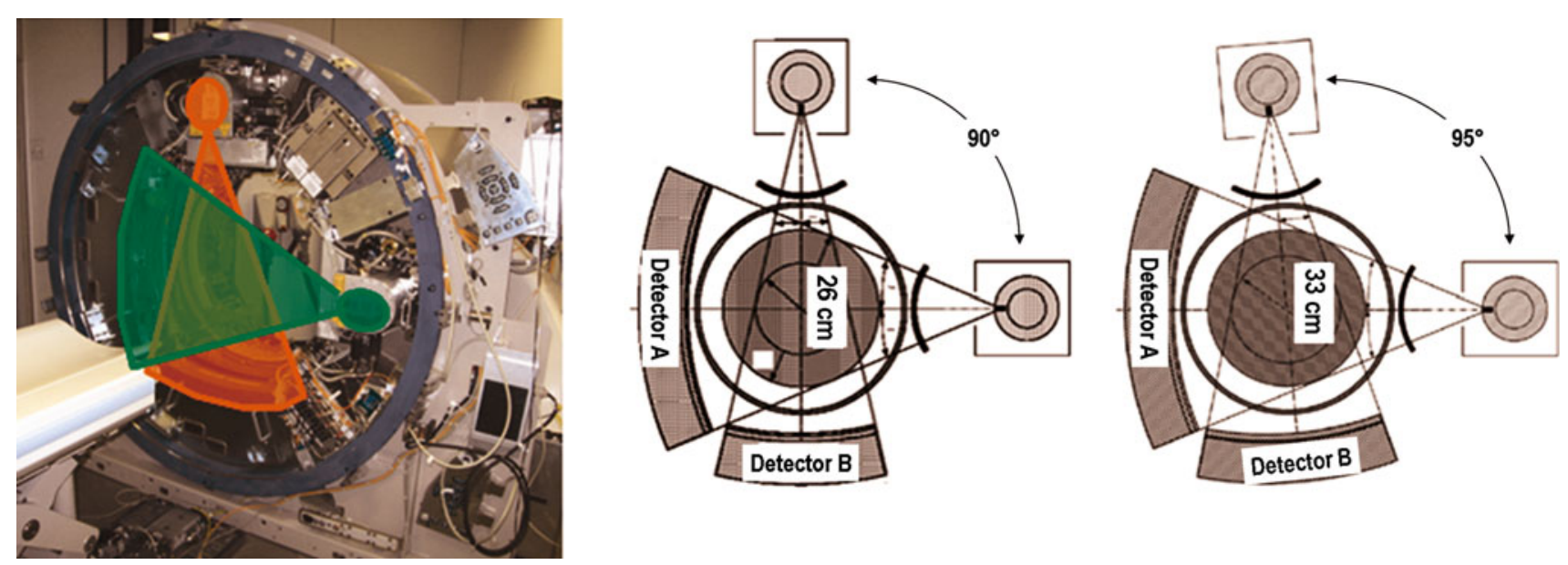

Fig. 8 Dual source CT-scanner with two independent measurement systems. First generation (center). The measurement systems are at an angle of $90^{\circ}$. Second generation (right). To enlarge the SFOV of detector B, the system angle was increased to $95^{\circ}$ 

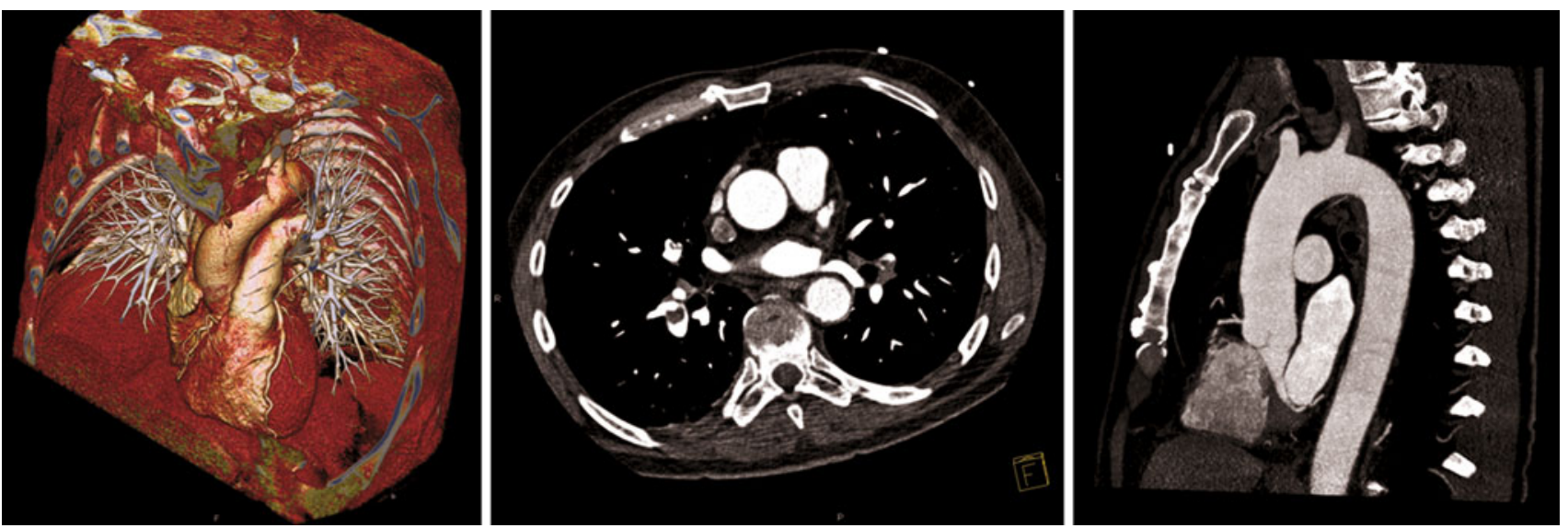

Fig. 9 ECG-triggered scan of the entire thorax using the dual source high-pitch spiral scan technique. Patient with bilateral pulmonary embolism. The total scan time to cover $269 \mathrm{~mm}$ was $0.6 \mathrm{~s}$, the

One option to provide CT scan data at different mean energies is to run one X-ray tube in a dual source CT at, e.g., $80 \mathrm{kV}$ and the other at $140 \mathrm{kV}$ (see Fig. 8). The scan parameters can be individually adjusted for both measurement systems, resulting in a flexible choice of scan modes. Optimization of the spectral separation is possible by introducing additional pre-filtration into the $140 \mathrm{kV}$ beam, e.g., by means of a filter that can be moved into the beam when needed and moved out for standard applications. An additional tin filter with a thickness of $0.4 \mathrm{~mm}$ shifts the mean energy of the $140-\mathrm{kV}$ spectrum behind the bow-tie filter from 69 to $89 \mathrm{keV}$ (see Fig. 10). The mean energy of the $80-\mathrm{kV}$ spectrum is $52 \mathrm{keV}$.

As a downside, dual energy evaluation with DSCT is restricted to the smaller central SFOV of detector B (26 resp. $33 \mathrm{~cm}$ diameter). Raw data based dual energy algorithms are difficult to realize, because high-energy and

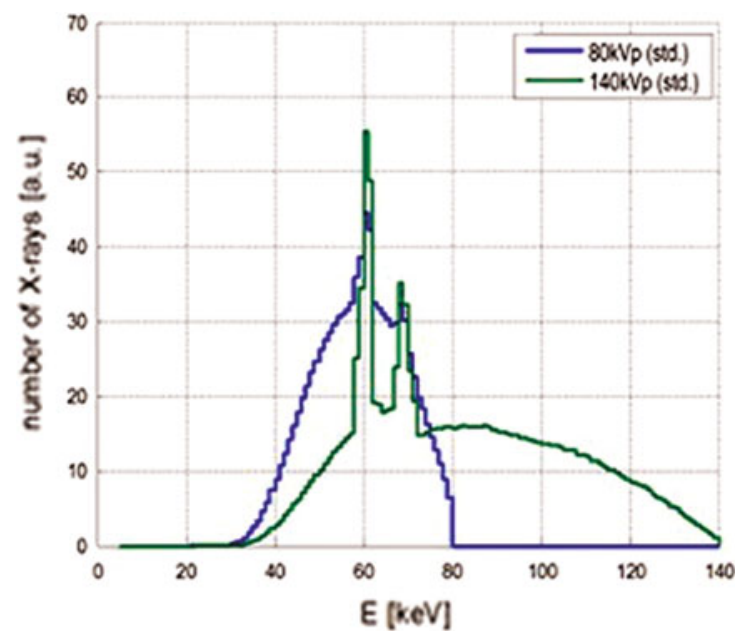

Fig. 10 Standard 80- and 140-kV spectra (left). 80-kV spectrum and $140-\mathrm{kV}$ spectrum with additional $0.4 \mathrm{~mm}$ tin pre-filtration (right). Note the shift of the $140-\mathrm{kV}$ spectrum to higher mean energy which radiation dose at $100 \mathrm{kV}$ and $370 \mathrm{mAs} /$ rot was $2.3 \mathrm{mSv}$ (courtesy of M. Lell, University of Erlangen, Germany)

low-energy projections are not simultaneously acquired at the same z-position. Dual energy algorithms are therefore image-based. Furthermore, images of moving objects show slightly different motion artifacts due to the $90^{\circ}$ offset between both measurement systems.

Another option to acquire dual energy data is fast switching of the X-ray tube voltage from one projection to the next in a single source CT ("fast kV-switching"), see Fig. 11.

Dual energy scans can be performed in the full SFOV of the respective CT scanner, and raw-data based evaluation approaches are possible. The nearly simultaneous acquisition of low-energy and high-energy projections prevents registration problems due to organ motion or contrast agent dynamics. However, as a down-side, spectral optimization by inserting additional pre-filters is not possible. Using current tube technology, only the tube voltage can be

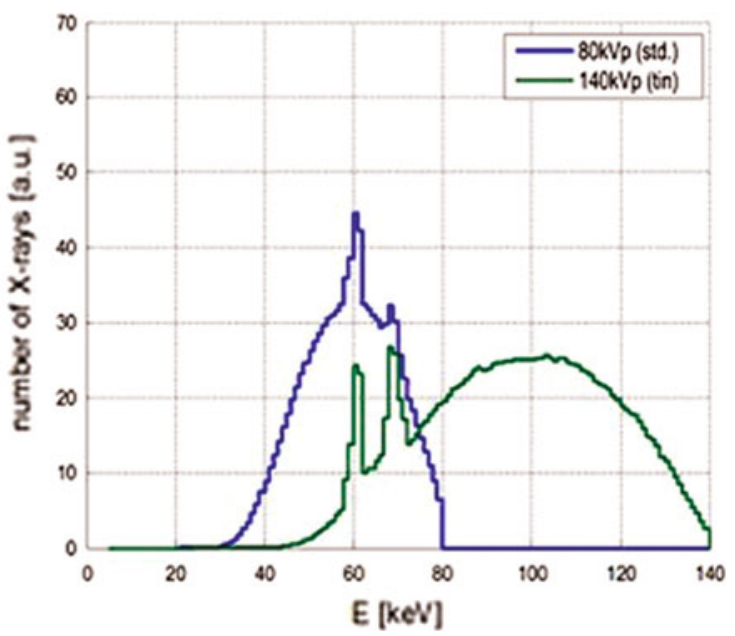

improves spectral separation (courtesy S. Kappler, Siemens Healthcare, Forchheim, Germany) 


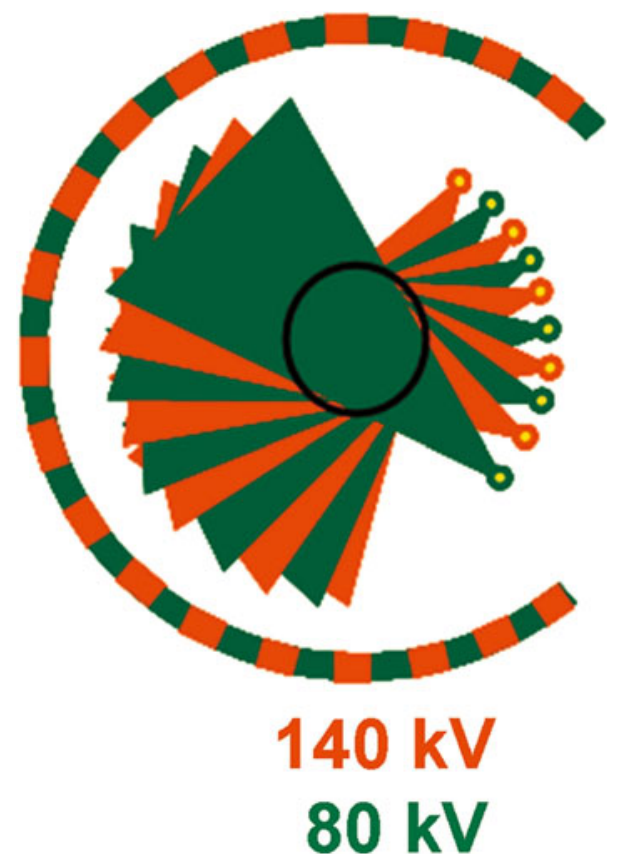

Fig. 11 Principle of fast $\mathrm{kV}$-switching to acquire dual energy CT data

switched between consecutive readings, but not the tube current. Equal dose at 80 and $140 \mathrm{kV}$ can only be achieved if each $80 \mathrm{kV}$ reading is a factor 2-4 longer than the corresponding $140 \mathrm{kV}$ reading. This reduces the number of available projections per rotation. Another drawback is the fixed and relatively high tube current that is required to obtain stable switching between both tube potentials (in terms of undershoots and overshoots of both voltage and current, stable shape and size of the focal spot). In one realization, a fixed tube current of $600 \mathrm{~mA}$ has to be applied [36, 37]. Radiation dose can only be adapted to the clinical application by variation of the spiral pitch and the rotation time.

Yet another option is the use of energy resolving detectors that allow the acquisition of spectral CT data with a single polychromatic X-ray spectrum. Pertinent examples are dual layer detectors consisting of two conventional scintillation detectors on top of each other, and direct converting photon counting detectors. So far, only prototype CT systems relying on both detector technologies have been realized. In particular photon-counting detectors, however, are a promising technology for future CTsystems.

Clinical applications of dual energy CT include tissue characterization, calcium quantification, calculation of pseudo-monochromatic images and quantification of the local blood volume in contrast-enhanced scans [38-41]. Figure 12 shows examples of dual energy applications.

\section{Alternative CT Designs}

A fifth-generation of CT scanners, the EBCT, uses a stationary X-ray tube and a stationary detector ring, thereby avoiding any mechanically moving parts. In these systems, an electron beam is emitted from a powerful electron gun and magnetically deflected to hit a semi-circular anode surrounding the patient. The magnetic deflection sweeps the electron beam over the target, thus generating an X-ray source that virtually rotates around the patient. Given the absence of mechanically moving parts, a sweep can be accomplished in as little as $50 \mathrm{~ms}$ (see Fig. 13).

Electron-beam CT systems were already introduced in 1984 as a non-invasive imaging modality for the diagnosis of coronary artery disease $[42,43]$. The technical principles were discussed in $[44,45]$. Despite its excellent temporal resolution, EBCT suffers from inherent disadvantages of the measurement principle, which have prevented a more wide-spread use in cardiology or general radiology. Due to the fourth-generation system geometry, the use of antiscatter collimator blades on the detector is not possible. As a consequence, image quality is degraded by scattered radiation. The housing of the electron gun limits the movement of the patient table and thus the scan range. Because of the fixed beam current of $640 \mathrm{~mA}$ in the commercially released EBCT-product, the exposure of only 32-64 mAs per slice was insufficient for larger patients.

In summary, the EBCT principle is not considered adequate either for state-of-the-art cardiac imaging or for general radiology applications, and has currently been abandoned.

Currently, newer experimental CT designs are being evaluated, such as inverse geometry CT systems [46, 47]. They use an X-ray tube with multiple distributed focal spots which sequentially illuminate a small detector to cover the examination object with several "sub-projections", see Fig. 14. These systems provide interesting advantages, such as the potential to shape the beam profile within a projection, thereby flexibly adapting the radiation dose to the size and shape of the patient. As a downside, they require very short acquisition times of the sub-projections to keep the total scan time within acceptable limits, and as a consequence short $\mathrm{X}$-ray pulses with very high tube output. Currently, tube technologies that could potentially be used in inverse geometry CT systems are not mature enough to enable routine clinical use.

Other, even more experimental $\mathrm{CT}$ designs, such as interior tomography $\left[48,53{ }^{\circ}\right]$ have not left the concept stage so far. Multisource interior tomography is intended to enable ultrafast imaging by irradiating a region of interest, such as the heart, in parallel with narrow X-ray beams defined by many source-detector pairs for data acquisition. This region of 

energy scan of a patient with metallic implants (top). The standard image shows severe metal artifacts (top left). Metal artifacts are significantly reduced by dual energy-based computation of pseudo-monoenergetic images at $120 \mathrm{keV}$ (top right). Dual source, dual gout (bottom). Uric acid crystals, which are a characteristic sign of gout, are identified and highlighted in Shaw Hospital, Hangzhou, China, and Clinical Innovation Center, Mayo Clinic Rochester, MN, USA)
Fig. 12 Dual source, dual energy scan of a patient with green (courtesy of Sir Run Run
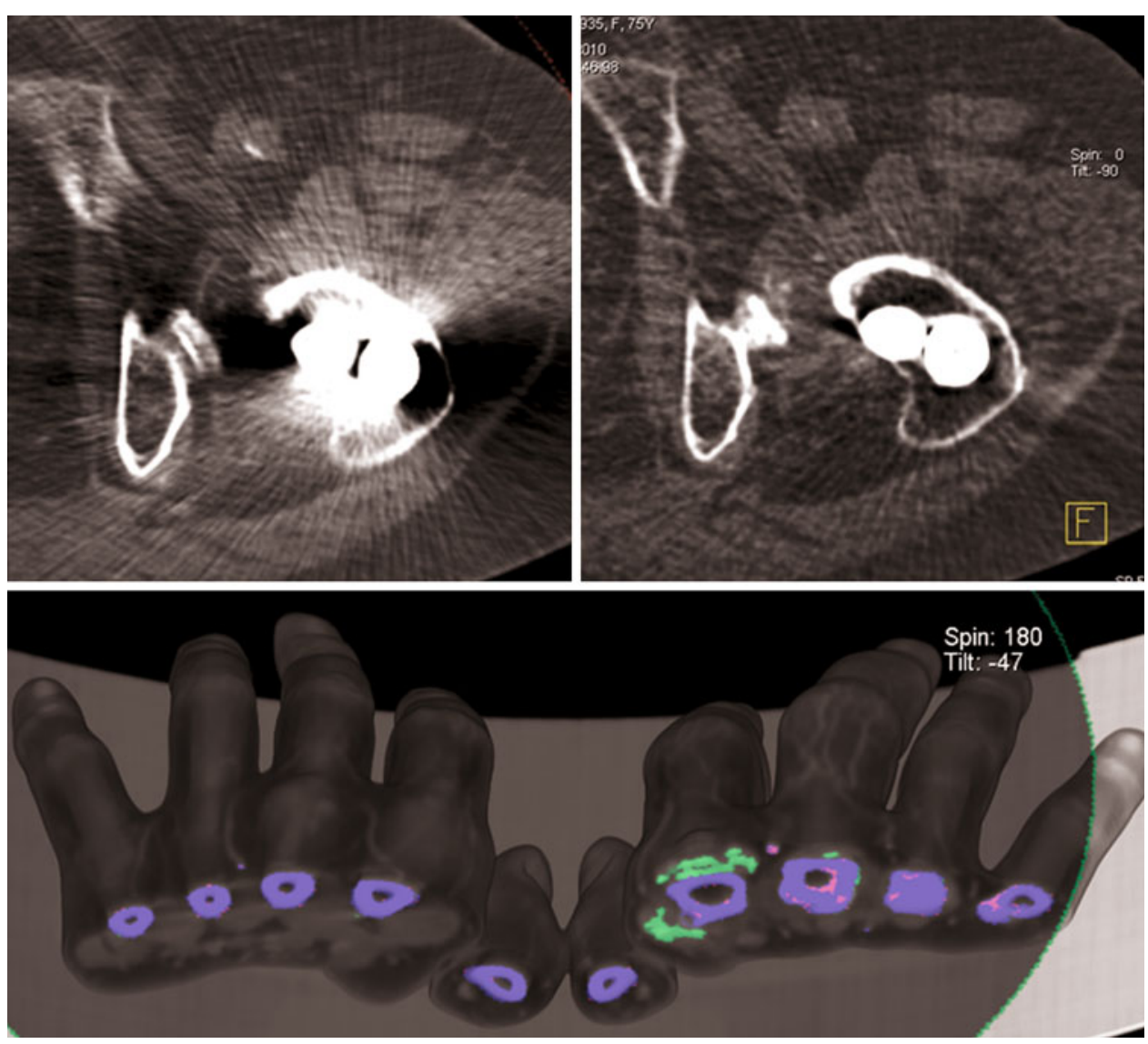

Fig. 13 Schematic illustration of an electron-beam CT (EBCT), a CT concept without mechanically moving parts. An electron beam is magnetically deflected to hit a semi-circular anode surrounding the patient (angular coverage $210^{\circ}$ ). The electron beam is swept over the target in 50-100 ms

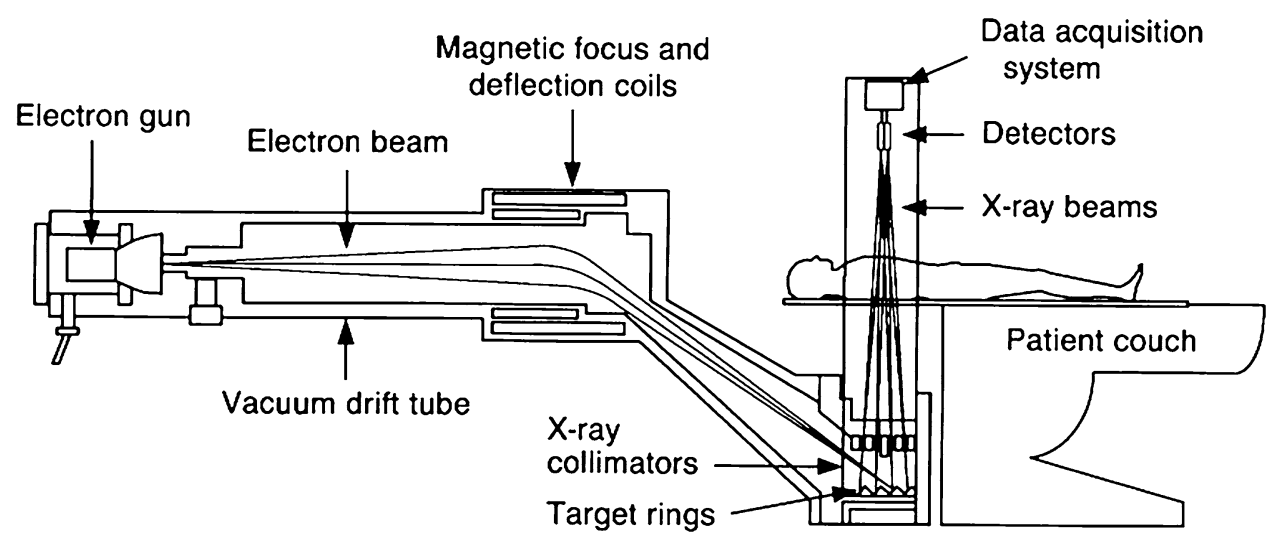

interest can be then reconstructed using the interior tomography approach.

All CT concepts discussed so far rely on the attenuation of $\mathrm{X}$-rays in the examined object and on measuring the intensity of the attenuated X-ray beam. Phase-contrast CT, on the other hand, is based on measuring the phase shift of the incoming X-rays, which are an electromagnetic wave, by the measurement object. Phase-contrast imaging has recently gained considerable attention in the scientific literature as a new method with potential applications in medical imaging, in particular after its successful implementation using low-brilliance sources [49]. For a typical photon energy of, e.g., $60 \mathrm{keV}$, the attenuation coefficient of relevant materials, such as water, is three orders of magnitude smaller than the phase shift. This suggests that phase contrast CT should be much more sensitive than absorption CT and could allow for a significantly reduced radiation dose to the patient. Unfortunately, this potential advantage of phase contrast CT can only be utilized at very high spatial resolution [50]. At the resolution level of today's medical CT, the relative performance of phase contrast CT is inferior to absorption CT. Imaging techniques already operating at higher spatial 
Fig. 14 Schematic illustration of a third-generation fan beam CT geometry (left) and an inverse CT geometry (right) (Courtesy of Dr. N. Pelc, Stanford University, USA)

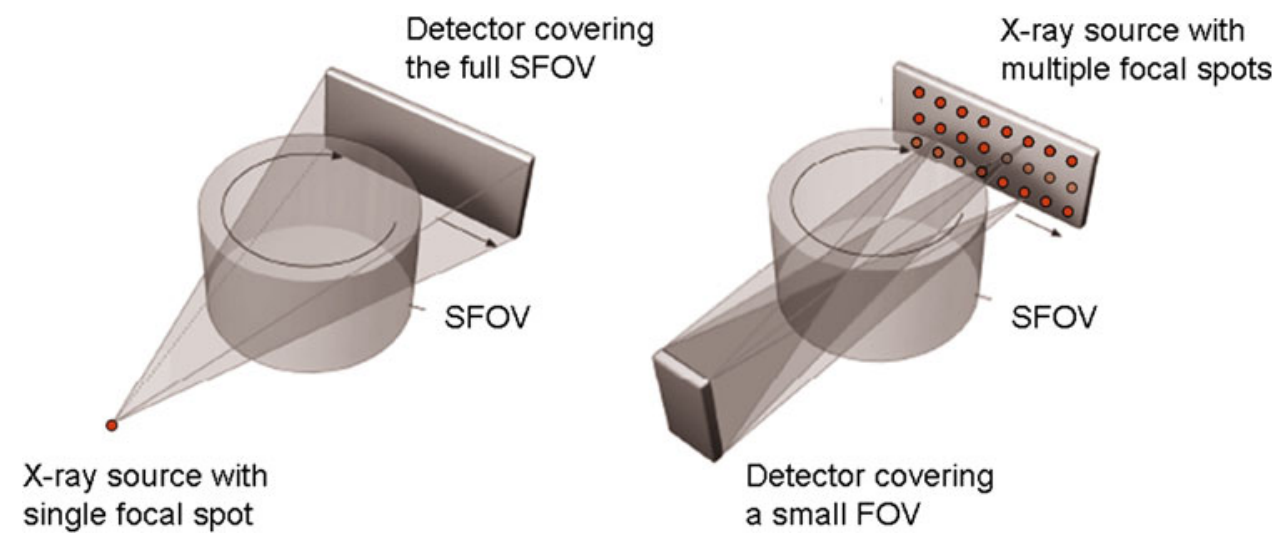

resolution, such as mammography, may benefit from phase contrast for some applications in terms of reduced patient dose at equal image quality [51].

\section{Conclusion}

We have reviewed the different system concepts of CT scanners from the introduction of CT in the early 1970s to modern MDCT, CT with area detectors, and dual source CT. Interestingly, despite all technological advances and improvements, all modern CT scanners are still based on the third-generation rotate-rotate geometry. Other system concepts, such as the fourth-generation CT scanners with rotating X-ray tube and stationary detector, or the fifthgeneration EBCT systems, have been abandoned because of severe practical drawbacks and limitations. Although several novel concepts for CT systems are currently being investigated, convincing approaches ready for routine clinical use and not relying on the classic "third-generation design" have not yet been presented-it will be interesting to witness the first real breakthrough in this aspect.

Disclosure T. Flohr is an employee of Siemens Healthcare, Forchheim, Germany.

\section{References}

Papers of particular interest, published recently, have been highlighted as:

•• Of major importance

1. Ambrose J, Hounsfield G. Computerized transverse axial tomography. Br J Radiol. 1973;46(542):148-9.

2. Crawford CR, King KF. Computed tomography scanning with simultaneous patient translation. Med Phys. 1990;17:967-82.

3. •• Kalender W, Seissler W, Klotz E, Vock P. Spiral volumetric CT with single-breath-hold technique, continuous transport and continuous scanner rotation. Radiology. 1990;176:181-3. This article lays the foundation of spiral CT scanning.

4. Kachelriess M, Ulzheimer S, Kalender W. ECG-correlated image reconstruction from subsecond multi-slice spiral CT scans of the heart. Med Phys. 2000;27:1881-902.

5. Ohnesorge B, Flohr T, Becker C, et al. Cardiac imaging by means of electro-cardiographically gated multisection spiral CT-initial experience. Radiology. 2000;217:564-71.

6. Klingenbeck-Regn K, Schaller S, Flohr T, et al. Subsecond multislice computed tomography: basics and applications. EJR. 1999;31:110-24.

7. McCollough $\mathrm{CH}$, Zink FE. Performance evaluation of a multislice CT system. Med Phys. 1999;26:2223-30.

8. $\mathrm{Hu} \mathrm{H}, \mathrm{He} \mathrm{HD}$, Foley WD, Fox SH. Four multidetector-row helical CT: image quality and volume coverage speed. Radiology. 2000;215:55-62.

9. Flohr T, Stierstorfer K, Bruder H, Simon J, Schaller S. New technical developments in multislice CT, part 1: approaching isotropic resolution with sub-mm 16-slice scanning. Röfo Fortschr Geb Rontgenstr Neuen Bildgeb Verfahr. 2002;174:839-45.

10. Flohr TG, Stierstorfer K, Ulzheimer S, et al. Image reconstruction and image quality evaluation for a 64-slice CT scanner with $z$ flying focal spot. Med Phys. 2005;32(8):2536-47.

11. Leber AW, Knez A, von Ziegler F, Becker A, Nikolaou K, Paul S, Wintersperger B, Reiser M, Becker CR, Steinbeck G, Boekstegers P. Quantification of obstructive and nonobstructive coronary lesions by 64-slice computed tomography. JACC. 2005; 46(1):147-54.

12. Raff GL, Gallagher MJ, O'Neill WW, Goldstein JA. Diagnostic accuracy of noninvasive coronary angiography using 64-slice spiral computed tomography. JACC. 2005;46(3):552-7.

13. Mori S, Endo M, Tsunoo T, Kandatsu S, Tanada S, Aradate H, et al. Physical performance evaluation of a 256-slice CT-scanner for four-dimensional imaging. Med Phys. 2004;31(6):1348-56.

14. Mori S, Endo M, Obata T, Tsunoo T, Susumu K, Tanada S. Properties of the prototype 256-row (cone beam) CT scanner. Eur Radiol. 2006;16(9):2100-8.

15. Mori S, Kondo C, Suzuki N, Hattori A, Kusakabe M, Endo M. Volumetric coronary angiography using the 256-detector row computed tomography scanner: comparison in vivo and in vitro with porcine models. Acta Radiol. 2006;47(2):186-91.

16. Kido T, Kurata A, Higashino H, Sugawara Y, Okayama H, Higaki J, Anno H, Katada K, Mori S, Tanada S, Endo M, Mochizuki T. Cardiac imaging using 256-detector row four-dimensional CT: preliminary clinical report. Radiat Med. 2007;25(1):38-44.

17. Rybicki FJ, Otero HJ, Steigner ML, et al. Initial evaluation of coronary images from 320-detector row computed tomography. Int J Cardiovasc Imaging. 2008;24(5):535-46. 
18. Hoe J, Toh KH. First experience with 320-row multidetector CT coronary angiography scanning with prospective electrocardiogram gating to reduce radiation dose. J Cardiovasc Comput Tomogr. 2009;3(4):257-61.

19. Dewey M, Zimmermann E, Deissenrieder F, Laule M, Dübel HP, Schlattmann P, Knebel F, Rutsch W, Hamm B. Noninvasive coronary angiography by 320-row computed tomography with lower radiation exposure and maintained diagnostic accuracy: comparison of results with cardiac catheterization in a head-tohead pilot investigation. Circulation. 2009;120(10):867-75.

20. Mori S, Obata T, Kato H, Kishimoto R, Kandatsu S, Tanada S, Endo M. Preliminary study: color map of hepatocellular carcinoma using dynamic contrast-enhanced 256-detector row CT. Eur J Radiol. 2007;62(2):308-10.

21. Flohr TG, Raupach R, Bruder H. Cardiac CT: how much can temporal resolution, spatial resolution, and volume coverage be improved? J Cardiovasc Comput Tomogr. 2009;3(3):143-52.

22. Engel KJ, Herrmann C, Zeitler G. X-ray scattering in single- and dual-soure CT. Med Phys. 2007;35(1):318-32.

23. Haberland U, Klotz E, Abolmaali N. Performance assessment of dynamic spiral scan modes with variable pitch for quantitative perfusion computed tomography. Invest Radiol. 2010;45(7): 378-86.

24. Robb R, Ritman E. High speed synchronous volume computed tomography of the heart. Radiology. 1979;133:655-61.

25. Ritman E, Kinsey J, Robb R, Gilbert B, Harris L, Wood E. Threedimensional imaging of heart, lungs, and circulation. Science. 1980;210:273-80.

26. Flohr TG, McCollough CH, Bruder H, Petersilka M, Gruber K, Süß C, Grasruck M, Stierstorfer K, Krauss B, Raupach R, Primak AN, Küttner A, Achenbach S, Becker C, Kopp A, Ohnesorge BM. First performance evaluation of a dual-source CT (DSCT) system. Eur Radiol. 2006;16(2):256-68.

27. Achenbach S, Ropers D, Kuettner A, Flohr T, Ohnesorge B, Bruder H, Theessen H, Karakaya M, Daniel WG, Bautz W, Kalender WA, Anders K. Contrast-enhanced coronary artery visualization by dual-source computed tomography-initial experience. Eur J Radiol. 2006;57(3):331-5.

28. Scheffel H, Alkadhi H, Plass A, et al. Accuracy of dual-source CT coronary angiography: first experience in a high pre-test probability population without heart rate control. Eur Radiol. 2006;16(12):2739-47.

29. Matt D, Scheffel H, Leschka S, Flohr TG, Marincek B, Kaufmann PA, Alkadhi H. Dual-source CT coronary angiography: image quality, mean heart rate, and heart rate variability. AJR Am J Roentgenol. 2007;189(3):567-73.

30. Leber AW, Johnson T, Becker A, von Ziegler F, Tittus J, Nikolaou K, Reiser M, Steinbeck G, Becker CR, Knez A. Diagnostic accuracy of dual-source multi-slice CT-coronary angiography in patients with an intermediate pretest likelihood for coronary artery disease. Eur Heart J. 2007;28(19):2354-60.

31. Ropers U, Ropers D, Pflederer T, et al. Influence of heart rate on the diagnostic accuracy of dual-source computed tomography coronary angiography. J Am Coll Cardiol. 2007;50(25):2393-8.

32. •• Petersilka M, Bruder H, Krauss B, Stierstorfer K, Flohr TG. Technical principles of dual source CT. Eur $J$ Radiol. 2008;68(3):362-8. This article gives an overview on technical principles, benefits, limitations and applications of dual source CT.

33. Flohr TG, Leng S, Yu L, Allmendinger T, Bruder H, Petersilka M, Eusemann CD, Stierstorfer K, Schmidt B, McCollough C. Dual-source spiral CT with pitch up to 3.2 and $75 \mathrm{~ms}$ temporal resolution: image reconstruction and assessment of image quality. Med Phys. 2009;36(12):5641-53.

34. Achenbach S, Marwan M, Ropers D, Schepis T, Pflederer T, Anders K, Kuettner A, Daniel WG, Uder M, Lell MM. Coronary computed tomography angiography with a consistent dose below
$1 \mathrm{mSv}$ using prospectively electrocardiogram-triggered highpitch spiral acquisition. Eur Heart J. 2010;31(3):340-6.

35. Leschka S, Stolzmann P, Desbiolles L, Baumueller S, Goetti R, Schertler T, Scheffel H, Plass A, Falk V, Feuchtner G, Marincek B, Alkadhi H. Diagnostic accuracy of high-pitch dual-source CT for the assessment of coronary stenoses: first experience. Eur Radiol. 2009;19(12):2896-903.

36. Lv P, Lin XZ, Li J, Li W, Chen K. Differentiation of small hepatic hemangioma from small hepatocellular carcinoma: recently introduced spectral CT method. Radiology. 2011;259(3):720-9.

37. Zhang D, Li X, Liu B. Objective characterization of GE discovery CT750 HD scanner: gemstone spectral imaging mode. Med Phys. 2011;38(3):1178-88.

38. Johnson TRC, Krauß B, Sedlmair M, Grasruck M, Bruder H, Morhard D, Fink C, Weckbach S, Lenhard M, Schmidt B, Flohr T, Reiser MF, Becker CR. Material differentiation by dual energy CT: initial experience. Eur Radiol. 2007;17(6):1510-7.

39. Primak AN, Fletcher JG, Vrtiska TJ, Dzyubak OP, Lieske JC, Jackson ME, Williams JC Jr, McCollough CH. Noninvasive differentiation of uric acid versus non-uric acid kidney stones using dual-energy CT. Acad Radiol. 2007;14(12):1441-7.

40. Scheffel H, Stolzmann P, Frauenfelder T, et al. Dual-energy contrast-enhanced computed tomography for the detection of urinary stone disease. Invest Radiol. 2007;42(12):823-9.

41. Graser A, Becker CR, Staehler M, Clevert DA, Macari M, Arndt N, Nikolaou K, Sommer W, Stief C, Reiser MF, Johnson TR. Single-phase dual-energy CT allows for characterization of renal masses as benign or malignant. Invest Radiol. 2010;45(7): $399-405$.

42. Agatston AS, Janowitz WR, Hildner FJ, Zusmer NR, Viamonte M, Detrano R. Quantification of coronary artery calcium using ultrafast computed tomography. JACC. 1990;15:827-32.

43. Budoff M, Georgiou D, Brody A, et al. Ultrafast computed tomography as a diagnostic modality in the detection of coronary artery disease: a multicenter study. Circulation. 1996;93:898-904.

44. McCollough $\mathrm{CH}$, Zink FE. The technical design and performance of ultrafast computed tomography. Radiol Clin North Am. 1994;32(3):521-36.

45. McCollough $\mathrm{CH}$, Zink FE, Morin R. Radiation dosimetry for electron beam CT. Radiology. 1994;192(3):637-43.

46. Schmidt TG, Fahrig R, Pelc NJ, Solomon EG. An inversegeometry volumetric CT system with a large-area scanned source: a feasibility study. Med Phys. 2004;31(9):2623-7.

47. de Man B, Basu S, Beque D, Claus B, Edic P, Iatrou M, et al. Multi-source inverse geometry CT : a new system concept for X-ray computed tomography. Proc SPIE Med Imaging 2007;6510: $65100 \mathrm{H}-2$.

48. Wang G, Yu H, Ye Y. A scheme for multisource interior tomography. Med Phys. 2009;36(8):3575-81.

49. Pfeiffer F, Weitkamp T, Bunk O, David C. Phase retrieval and differential phase-contrast imaging with low-brilliance X-ray sources. Nat Phys. 2006;2:258-61.

50. Raupach R, Flohr TG. Analytical evaluation of the signal and noise propagation in X-ray differential phase-contrast computed tomography. Phys Med Biol. 2011;56:2219-44.

51. Raupach R, Flohr TG. Performance evaluation of X-ray differential phase contrast computed tomography (PCT) with respect to medical imaging. Med Phys. 2012;39(8):4761-74.

52. Mori S, Endo M, Nishizawa K, et al. Comparison of patient doses in 256-slice $\mathrm{CT}$ and 16-slice CT scanners. $\mathrm{Br} \mathrm{J}$ Radiol. 2006;79(937):56-61.

53. • Wang G, Yu H, de Man B. An outlook on X-ray CT research and development. Med Phys. 2008;35(3):1051-64. This article reviews current research directions in computed tomography and may serve as an overview. 